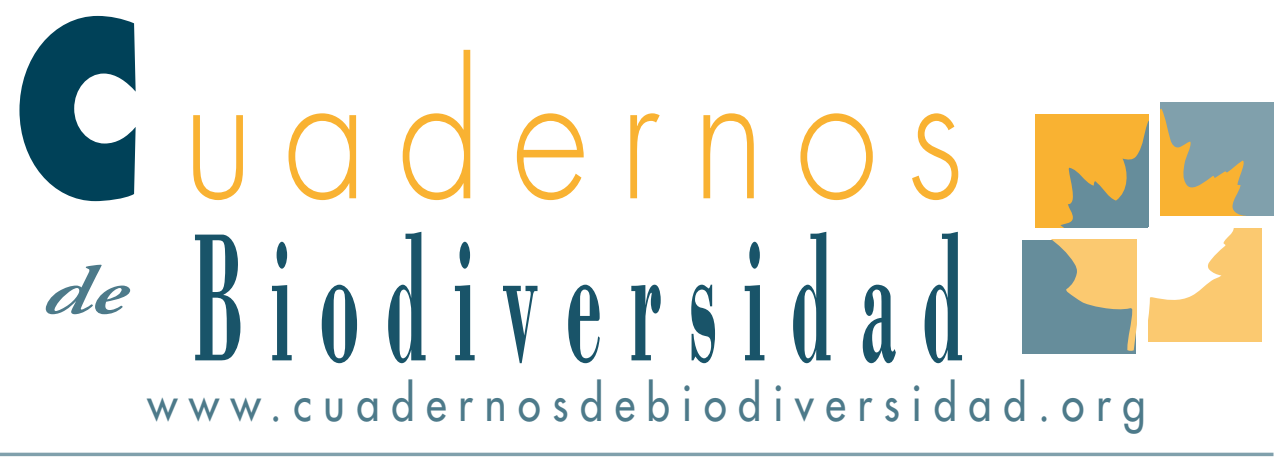

\title{
Rhizaspidiotus donacis (Hemiptera; Diaspididae); agente con licencia para controlar. Primeros resultados del plan de control biológico del carrizo gigante en Estados Unidos
}

\section{E. Cortés ${ }^{1}$, M.A. Marcos-García \& J. Goolsby ${ }^{2}$}

i Centro Iberoamericano de Biodiversidad (CIBIO), Universidad de Alicante, España. ELENA.CORTES@UA.ES MARCOS@UA.ES

2 United States Department Of Agriculture, Agricultural Research Service. JOHN.GOOLSBY@ARS.USDA.GOV

\section{ABSTRACT}

More than ten years ago, a biological control plan was initiated by Department of Agriculture of the United States (USDA) in order to find a solution for counteracting the invasion of the Red Giant: Arundo donax Linnaeus (1753) in riparian ecosystems of USA and México. This invasive plant is originating serious environmental and economic problems in these natural areas as the displacement of native plant species, decreasing in its associate fauna biodiversity and an important water consume.
An insect complex was considered as potential biological control agents against this grass, between them, Rhizaspidiotus donacis Leonardi (1920) (Hemiptera; Diaspididae) was one of the most encouraging agents due to this insect exerts a significant impact on both, aerial and underground parts of the plant. Nowadays, the releases of this monofagous insect have been accepted and carried out along Río Grande, one of the most affected natural areas. The settlement and reproduction of $R$. donacis were reported with good preliminary observations about the impact of the insect in the invasive areas. 


\section{KEY WORDS:}

Biological control, Invasive plant, Rhizaspidiotus donacis, Impact, Arundo donax

\section{INTRODUCCIÓN}

A finales de la década de los 90 se iniciaba desde el Departamento de Agricultura de los EEUU (USDA) un ambicioso proyecto para frenar la invasión de una gramínea invasora, Arundo donax L. (Poaceae; Arundinoideae) en ecosistemas ribereños del Suroeste de EEUU y Norte de México mediante técnicas de control biológico (CORTÉS et al. 2009) Esta iniciativa surge ante la imposibilidad de controlar el crecimiento de Arundo donax mediante métodos químicos y mecánicos. Una de las áreas más fuertemente afectadas es la cuenca de río Grande, que ejerce de frontera entre ambos países y que actualmente ya cuenta con casi una tercera parte de sus $3000 \mathrm{~km}$ de longitud invadidos por $A$. donax. En las zonas ribereńas donde crece en importantes densidades, esta planta de gran porte erosiona los cauces, desplaza a la vegetación nativa (especies pertenecientes a los géneros Salix, Populus o Bacharis) y con ello, a su fauna asociada que en muchas ocasiones se encuentra amenazada como las aves autóctonas de pequeño tamaño Vireo bellii pusillus (Coues, 1866) y Empidonax traillii extimus (Phillips, 1948), entre otras (ZEMBAL, 1986, 1990; BELL, 1997).

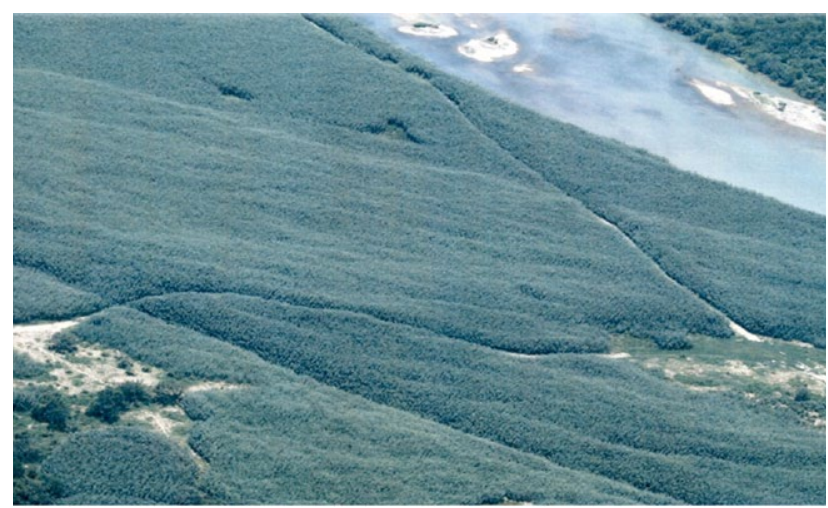

Figura 1. Río Grande a su paso por Del Rio (suroeste de Texas). Se observa el cauce invadido por Arundo donax.
En 2004, a través de un convenio de colaboración se incluye la participación del CIBIO (Centro Iberoamericano para el estudio de la Biodiversidad) en este proyecto de Control Biológico mediante el desarrollo de una tesis doctoral con el fin de completar el conocimiento sobre la biología y el nivel de impacto que un agente de control biológico preseleccionado ejercía sobre la planta rizomatosa.

Este agente es un pequeño insecto perteneciente a la familia Diaspididae, lo que vulgarmente denominamos "cochinillas con escudo". La especie es Rhizaspidiotus donacis (Leonardi) (Hemiptera; Diaspididae), y al igual que otros insectos considerados en este plan de control, fue evaluado para evaluar su nivel de especificidad e impacto. Los individuos de $R$. donacis se ubican en los rizomas de la planta y en la inserción de los brotes laterales al eje principal de la misma. Desde aquí, ejercen un daño directo mediante su acción fitófaga que se pone de manifiesto cuando se enlentece significativamente el crecimiento de estas estructuras (casi a la mitad) una vez que se encuentran infestadas por este diaspídido, produciéndose un impacto notable tanto en la parte aérea de la planta (CORTÉS et al. 2011a) como en las estructuras subterráneas (CORTÉS et al. 2011b), principal método de dispersión en esta especie vegetal.

Estos resultados se obtuvieron gracias al trabajo de campo desarrollado en el contexto de la citada tesis doctoral, y que fue llevado a cabo en diversas poblaciones de $A$. donax ubicadas en la provincia de Alicante, por formar parte del área geográfica nativa de distribución, tanto de la gramínea objetivo de control, como del insecto fitófago considerado.

Además de estos resultados sobre su control, también se realizaron estudios sobre la biología, ciclo de vida (CORTÉS \& MARCOS-GARCÍA, 2012) y monofagia del insecto (GOOLSBY et al. 2009), conocimiento previo imprescindible para su definitiva consideración como agente de control biológico. 


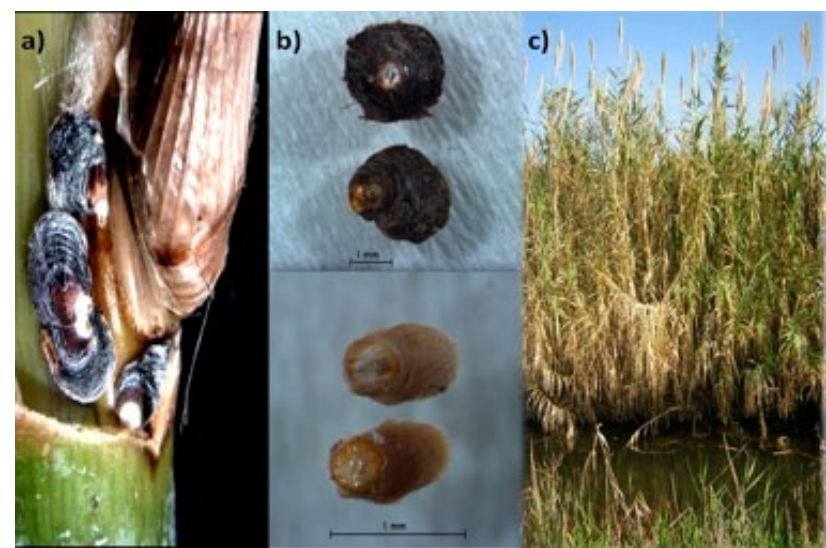

Figura 2. a. Insecto alimentándose en la base de los tallos. b. Escudos protectores característicos de estos insectos (hembras, imagen superior, machos imagen inferior). c. Grupo de cañas.

\section{EXPECTATIVAS Y SITUACIÓN ACTUAL}

La esperanza está depositada en el uso de un complejo de insectos, formado por dos especies de dípteros (un cecidómido y un clorópido), un himenóptero perteneciente a la familia Eurytomidae y el citado diaspídido. El principal objetivo es lograr que todos estos insectos sean liberados en campo para maximizar el impacto sobre la planta invasora, habiéndose descrito efectos sinérgicos con el uso combinado de alguno de estos agentes. Las larvas del himenóptero Tetramesa romana (Walker, 1873), son barrenadoras del tallo de la caña, induciendo con su acción la formación agallas que pueden favorecer la emergencia de nuevos brotes laterales. Estos brotes constituyen una de las estructuras preferidas como lugar de asentamiento para las ninfas de Rhizaspidiotus donacis, que ven ampliada así la disponibilidad de lugares con las características necesarias para ser colonizados por estos diaspídidos.

Hace unos pocos años fue aceptada la suelta de $T$. romana y actualmente se combinan las liberaciones de este himenóptero con las de $R$. donacis en los lugares de invasión. Para que dichas sueltas puedan ser llevadas a cabo por la institución peticionaria (USDA), los agentes de control, insectos en este caso, que se pretenden liberar, deben cumplir con una serie de requisitos que garanticen que no se ocasionarán nuevos desequilibrios en los ecosistemas. Para ello, deben presentarse pruebas de especificidad realizadas para cada uno de los potenciales agentes de control al 'Technical Advisory Group' (TAG) para que una vez revisada pueda ser aprobada la suelta.

ElTAG para el control biológico de plantas invasoras fue creado en 1987 y está formado por 15 agencias gubernamentales que representan a Estados Unidos, México y Canadá.

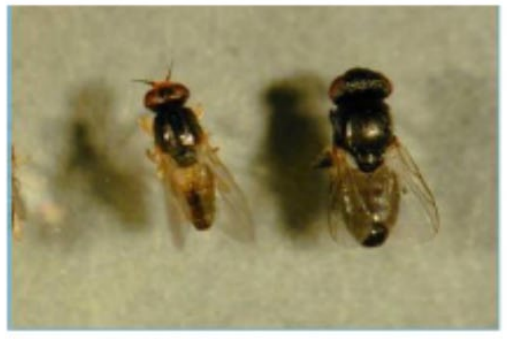

Cryptonevra spp.

Diptera:Chloropidae

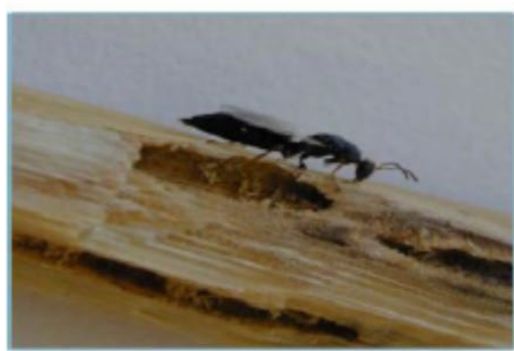

Tetramesa romana Hymenoptera: Eurytomidae
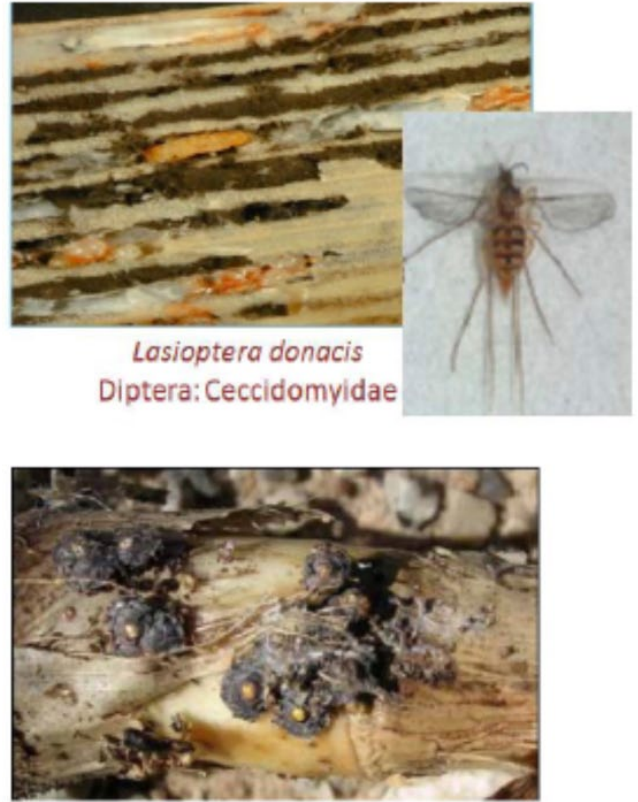

Rhizaspidiotus donacis Hemíptera:Diaspididae
Figura 3. Complejo de insectos que componen el grupo de agentes de control biológico de Arundo donax. 
Cuando una petición es enviada y revisada por el TAG, este organismo puede recomendar la suelta del agente, lo cual lleva consigo una nueva petición de un permiso de aplicación por parte de la institución solicitante al 'Animal \& Plant Health Inspection Service (APHIS) Plant Protection and Quarantine' (PPQ) quienes llevan a cabo una evaluación medioambiental (EA). Si el EA obtiene una valoración de 'No existe un impacto significativo', entonces el APHIS-PPQ concede el permiso. Todo este proceso asegura que únicamente sean liberados los agentes específicos, reduciendo así enormemente los riesgos inherentes que el desarrollo de un plan de control biológico conlleva cuando se aplican en un nuevo lugar.

Para llevar a cabo las sueltas del diaspídido, se realizaron recogidas de material (rizomas infestados) en varios puntos dentro de su área nativa de distribución (Rivesaltes en el río Roboul próximo a Perpignan al sureste francés, y desde río Seco a su paso por Villafranqueza en Alicante, al sureste de la costa Mediterránea española). El material recolectado fue enviado a los laboratorios y cuarentenas del USDA-APHIS-PPQ en Texas donde fue procesado. Las hembras maduras de dichos rizomas fueron individualizadas en cápsulas y una vez que produjeron ninfas, éstas fueron transferidas a nuevas cápsulas y transportadas a las áreas de invasión, donde eran liberadas tanto sobre la parte aérea de las cañas como sobre rizomas que quedaban a medio enterrar.

En la ciudad de Del Rio, al suroeste del Estado Texano, un total de 634.947 ninfas fueron liberadas desde Enero hasta Abril de 2011 en las densas poblaciones de $A$. donax originadas en el cauce de río Grande a su paso por esta localidad. Dos meses después de llevar a cabo estas sueltas ya se ha registrado un gran número de escudos vacíos pertenecientes a machos de esta especie, hecho que evidencia la emergencia de dichos machos alados en su fase adulta (GOOLSBY et al. 2011).

Cinco meses después de ejecutarse las sueltas, se iniciaron inspecciones de las cañas infestadas y se registraron hembras adultas, observándose también la emergencia de las nuevas ninfas en Julio.
Resultados similares se observaron en estudios desarrollados en invernaderos en Texas y Juitepec (Cuernavaca), Mexico (M. Martínez-Jiménez, datos no publicados).

Estas observaciones resultaron coincidentes con los estudios sobre su biología desarrollados en las cuarentenas (MORAN \& GOOLSBY, 2010). Aunque aún preliminares, estos datos indican que la primera iniciativa de uso de un diaspídido como agente de control biológico para el control de una planta invasora, puede contribuir significativamente a una solución definitiva. El impacto ejercido en la actualidad por el insecto en estas áreas naturales de Estados Unidos, puede calificarse de moderado, con la esperanza de que se vea mejorado en las próximas generaciones y potenciado una vez que interactúe con T. romana. Al mismo tiempo, la adaptación del diaspídido a las áreas de invasión, supone el primer registro del establecimiento de $R$. donacis en Norte América y probada su monofagia, sin riesgo alguno de provocar nuevos desequilibrios ambientales.

\section{REFERENCIAS}

BELL, G. P. 1997. Ecology and management of Arundo donax, and approaches to riparian habitat restoration in Southern California, pp. 103-113. In J. H.Brock, M. Wade, P. Pysek, and D. Green [eds.], Plant Invasions: Studies from North America and Europe. Blackhuys Publishers, Leiden, Netherlands.

CORTÉS, E., M. A. MARCOS - GARCÍA \& J. GOOLSBY. 2009. Buscando el antídoto natural en la lucha contra el "carrizo gigante". Cuadernos de Biodiversidad 29: 20-24.

CORTÉS, E., J. GOOLSBY, P. MORAN \& M.A. MARCOSGARCÍA. 2011a. The effect of the armored scale, Rhizaspidiotus donacis (Leonardi) (Hemiptera;Diaspididae), on shoot growth of the invasive plant Arundo donax (Poaceae;Arundinoideae), Biocontrol Science and Techno$\log y 21: 5,535-545$.

CORTÉS, E., A. A. KIRK, J. A. GOOLSBY, P. J. MORAN, A. E. RACELIS \& M. A. MARCOS-GARCÍA. 2011 b. Impact of the Arundo scale Rhizaspidiotus donacis (Leonardi) (Hemiptera; Diaspididae) on the weight of Arundo donax L. (Poaceae; Arundinoideae) rhizomes in Languedoc southern France and Mediterranean Spain. Biocontrol Science and Technology 21: 1369-1373. 
GOOLSBY, J. A., A. A. KIRK, P. J. MORAN, A. E. RACELIS, J. J. ADAMCZYK, E. CORTÉS, M.A. MARCOSGARCÍA, M.J. JIMENEZ MARTÍNEZ,K. R. SUMMY, M. A. CIOMPERLIK \& D. P. A. SANDS. 2011. Establishment of the Armored Scale, Rhizaspidiotus donacis, a Biological Control Agent of Arundo donax. Southwestern Entomolologist 36: 373-374.

MORAN, P. J., \& J. A. GOOLSBY. 2010. Biology of the armored scale Rhizaspidiotus donacis (Hemiptera: Diaspididae), a candidate agent for biological control of giant reed. Annals of the Entomological Society of America 103: 252-263.
ZEMBAL, R. 1986. The Least Bell's Vireo in the Prado Basin and environs, 1985. Unpublished report, U.S. Fish and Wildlife service, Laguna Niguel, CA.

ZEMBAL, R. 1990. Riparian habitat and breeding birds along the Santa Margarita and Santa Ana Rivers of southern California. In: A.A.Schoenherr (ed.), Endangered plant communities of southern California, pp. 98-114. Southern California Botanists, Special Publ. No. 3, Fullerton, CA. 